\title{
Phenome-wide association study of TTR and RBP4 genes in 361,194 individuals reveals novel insights in the genetics of hereditary and wildtype transthyretin amyloidoses
}

\author{
Antonella De Lillo ${ }^{1}$ Flavio De Angelis ${ }^{1} \cdot$ Marco Di Girolamo $^{2} \cdot$ Marco Luigetti $^{3}$ - Sabrina Frusconi ${ }^{4}$. \\ Dario Manfellotto ${ }^{2} \cdot$ Maria Fuciarelli ${ }^{1} \cdot$ Renato Polimanti ${ }^{5,6}$
}

Received: 12 August 2019 / Accepted: 22 October 2019/ Published online: 29 October 2019

(c) Springer-Verlag GmbH Germany, part of Springer Nature 2019

\begin{abstract}
Transthyretin (TTR) gene has a causal role in a hereditary form of amyloidosis (ATTRm) and is potentially involved in the risk of wild-type transthyretin amyloidosis (ATTRwt). To understand the genetics of ATTRm and ATTRwt, we conducted a phenome-wide association study of TTR gene in 361,194 participants of European descent testing coding and non-coding variants. Among the 382 clinically relevant phenotypes tested, TTR non-coding variants were associated with 26 phenotypic traits after multiple testing correction. These included signs related to both ATTRm and ATTRwt such as chronic ischaemic heart disease ( $\left(r s 140226130, p=2.00 \times 10^{-6}\right)$, heart failure (rs73956431, $\left.p=2.74 \times 10^{-6}\right)$, atrial fibrillation $\left(\mathrm{rs} 10163755, p=4.63 \times 10^{-6}\right)$, dysphagia $\left(\mathrm{rs} 2949506, p=3.95 \times 10^{-6}\right)$, intestine diseases $\left(\mathrm{rs} 970866, p=7.14 \times 10^{-6}\right.$ ) and anxiety (rs554521234, $p=8.85 \times 10^{-6}$ ). Consistent results were observed for TTR disease-causing mutation Val122Ile (rs76992529) with respect to carpal tunnel syndrome $\left(p=6.41 \times 10^{-6}\right)$ and mononeuropathies of upper limbs $\left(p=1.22 \times 10^{-5}\right)$. Sex differences were also observed in line with ATTRm and ATTRwt epidemiology. Additionally, we explored possible modifier genes related to TTR function, observing convergent associations of RBP4 variants with the clinical phenotypes associated with TTR locus. In conclusion, we provide novel insights regarding the molecular basis of ATTRm and ATTRwt based on large-scale cohort, expanding our understanding of the phenotypic spectrum associated with TTR gene variation.
\end{abstract}

Electronic supplementary material The online version of this article (https://doi.org/10.1007/s00439-019-02078-6) contains supplementary material, which is available to authorized users.

Renato Polimanti

renato.polimanti@yale.edu

1 Department of Biology, University of Rome Tor Vergata, Rome, Italy

2 Clinical Pathophysiology Center, Fatebenefratelli Foundation-'San Giovanni Calibita' Fatebenefratelli Hospital, Rome, Italy

3 Fondazione Policlinico Universitario A. Gemelli IRCCS, UOC Neurologia, Università Cattolica del Sacro Cuore, Rome, Italy

4 Genetic Diagnostics Unit, Laboratory Department, Careggi University Hospital, Florence, Italy

5 Department of Psychiatry, Yale University School of Medicine, West Haven, CT, USA

6 VA CT Healthcare Center, VA CT 116A2, 950 Campbell Avenue, West Haven, CT 06516, USA

\section{Introduction}

Transthyretin (TTR) misfolding and the consequent amyloid formation and deposition lead to two form of amyloidoses: a hereditary (or mutant) form (ATTRm) caused by more than 130 TTR gene coding mutations (Conceicao et al. 2019; Plante-Bordeneuve and Said 2011), and a senile systemic form that is due to the misfolding of wild type protein (wild-type transthyretin amyloidosis, ATTRwt; i.e. no TTR coding mutation present in the individuals affected by ATTRwt; (Westermark et al. 1990). ATTRm is a life-threatening disorder that affects several tissues (e.g. brain, autonomic and peripheral nerves, heart, and gastrointestinal (GI) tract) and is characterized by a complex genotype-phenotype correlation (Conceicao 2012; Hellman et al. 2008; Palaninathan 2012; Parman et al. 2016). Despite TTR coding mutations are the cause of the disease, the clinical variability of the ATTRm is likely due to several other factors (Alves-Ferreira et al. 2018; Iorio et al. 2015, 2017a; b; Polimanti et al. 2014). Differently from ATTRm, patients affected by ATTRwt do not present a coding mutation in TTR gene and the main symptoms include 
cardiac failure and carpal tunnel syndrome (Connors et al. 2011; Kyle and Gertz 1995; Maceira et al. 2005; Nakagawa et al. 2016; Pitkanen et al. 1984). TTR non-coding variation via its regulatory function seems to have a role in the phenotypic heterogeneity of ATTRm and to play a role in the pathogenesis of ATTRwt (Iorio et al. 2017b; Polimanti et al. 2013, 2019; Sikora et al. 2015). Furthermore, variation in other genes encoding for protein products that interact with TTR tetramer seems to modulate the pathogenetic processes involved in the TTR fibrils formation and, consequently, in the course of the disease (Santos et al. 2016; Soares et al. 2005; White and Kelly 2001). Genome-wide datasets generated from cohorts including hundred thousand participants can be leveraged to disentangle complex genotype-phenotype associations. In particular, phenome-wide association studies (PheWAS) can permit us to detect novel associations with respect to known risk loci with respect to a wide range of phenotypes (Bush et al. 2016; Denny et al. 2013; Polimanti et al. 2016). In the present study, we explored the phenotypic spectrum associated with the coding and non-coding variants of TTR gene, also investigating potential modifier loci that could modulate TTR amyloidogenic process. To date, the vast majority of the studies that investigated the genetics of ATTRm and ATTRwt have been conducted on cohorts with a limited sample size due to the low disease prevalence (Iorio et al. 2015, 2017b; Sikora et al. 2015). ATTRm and ATTRwt signs are poorly recognized and the correct diagnosis in sporadic cases is usually established several years from the onset of the symptoms (Ando et al. 2013). This consistently limited the ability to collect large informative cohorts needed to investigate the genetics of ATTRm and ATTRwt. To date, it is possible to investigate cohorts including hundred thousand participants to explore the association of genetic variation with clinically relevant phenotypes. Accordingly, we conducted a PheWAS in 361,194 participants of European descent available from the UK Biobank with respect to 382 clinically relevant traits to investigate genetic variation potentially involved in TTRrelated pathogenic processes. The results obtained support that non-coding variants located in TTR gene and other diseasemodifying loci are associated with phenotypic traits related to ATTRm and ATTRwt. On the basis of the current findings, we hypothesize that non-coding variation is involved (1) in the variability of the phenotypic presentation of carriers of TTR coding mutations; and (2) in the increased risk of ATTRwt in non-carriers of $T T R$ coding mutations.

\section{Materials and methods}

This study was conducted leveraging genome-wide association data generated from the UK Biobank (361,194 participants and 382 phenotypes). These datasets was used to explore the phenotypic spectrum associated with common genetic variants located in TTR gene and its surrounding regions. Additionally, we tested whether the protein product of TTR gene showed strong evidence of interaction with other proteins and verified whether genetic variants located in the encoding gene of these proteins were associated with the same phenotypic traits identified in TTR association analysis. A schematic workflow summarizing the analyses conducted is reported in Supplementary File 1.

\section{Genome-wide datasets}

The dataset used for the analysis was derived from the UK Biobank. This is an open access resource available to investigate a wide range of serious and life-threatening illnesses (Allen et al. 2014). This project has recruited more than 500,000 people assessed for a wide range of phenotypic information, also including clinically relevant phenotypes. Genetic data are available for the whole cohort and were used to generate genome-wide association datasets that can be used to explore the genetics of human diseases and traits. These genome-wide datasets used in the present study were generated from the analysis of 361,194 unrelated participants of European descent including 194,174 women and 167,020 men. The association analysis for all phenotypes was conducted using appropriate regression models available in Hail (available at https://github.com/hail-is/hail) including the first 20 ancestry principal components, sex, age, age ${ }^{2}$, sex $\times$ age, and sex $\times$ age $^{2}$ as covariates. The principal components included in the regression model were generated by the UK Biobank investigators using fastPCA algorithm and considering unrelated subjects and genetic markers pruned for linkage disequilibrium (Bycroft et al. 2018). Details regarding QC criteria, GWAS methods, and the original data are available at https://github.com/Neale lab/UK_Biobank_GWAS/tree/master/imputed-v2-gwas.

\section{Clinically relevant phenotypes}

Since our goal was to investigate phenotypes related to the pathogenesis of ATTRm and ATTRwt, we focused on clinically relevant traits available in the UK Biobank. We considered ICD-10 [International Classification of Disease, 10th Revision; (Denny 2012; Wei and Denny 2015)] codes and clinical endpoints derived by the FinnGen project (information available at https://www.finngen.fi/en). ICD-10 codes are a specific terminology that include diseases, signs, symptoms, and procedure codes maintained by the World Health Organization (WHO), and they typically are used to billing data (Denny 2012). The FinnGen project comes from a collaboration among Finnish universities, biobanks, hospital districts, and several international pharmaceutical companies to increase knowledge about human diseases. FinnGen clinical endpoints (available at https://www.finngen.fi/en/ 
researchers/clinical-endpoints) were developed to conduct genome-wide investigations of phenotypic traits assessed across several national health registers. To remove phenotypes not informative due to a lack of statistical power, we investigated phenotypic traits with a number of cases greater than 1000 individuals. The full list of the phenotypes investigated, and their corresponding sample size is reported in the Supplementary File 2. Additionally, we also conducted sex-stratified analysis to investigate the known differences between sexes in the epidemiology of ATTRm and ATTRwt. A total of 382, 240, and 225 clinically relevant phenotypes were investigated in the total sample and in the sex-stratified analysis (female- and male-specific analyses, respectively).

\section{Data analysis}

We considered a total of 382 clinically relevant phenotypes (sex-stratified analysis: 240 for female-specific PheWAS and 225 for male-specific PheWAS) and investigated variants located in $T T R$ gene considering a $4-\mathrm{Mb}$ region (NC_000018.9: 27,171,000-31,171,500) including the genic locus (NM_000371; 7257 bp) and the surrounding regions ( $\pm \sim 2 \mathrm{Mb}$ from the transcription start/end sites) (Table 1). We initially applied a filter to the variants investigated on the basis of their minor allele frequency (MAF $>1 \%$ ). Additionally, as recommended in the original UK Biobank analysis (information available at http://www.nealelab.is/ blog/2017/9/11/details-and-considerations-of-the-uk-bioba nk-gwas), we considered high-confidence association results generated from variants with at least 25 minor alleles in the smaller group (case or control). A total of 12,719, 12,710, and 12,711 high-confidence TTR variants that were investigated in the overall-sample and in the sex-stratified analyses (male- and female-specific, respectively). False Discovery Rate (FDR; (Benjamini and Hochberg 1995) at 10\% was applied as significance threshold for multiple testing correction accounting for the number of variants and the number of traits tested. This significance threshold was selected on the basis of the correlation expected among the variants and the phenotypes tested. To estimate the independent association signals within the genomic region tested, PLINK 1.09 (Chang et al. 2015) was used to perform linkage disequilibrium (LD) clumping considering a $0.1 R^{2}$ cut-off within a 500-kb window.

STRING v.11.0 (Szklarczyk et al. 2019) was used to identify protein interaction with $T T R$, considering experiments, co-expression, co-occurrence, gene fusion, and neighbourhood as active sources and a confidence score higher than 0.9 . With respect to proteins identified, we investigated variants included in coding, non-coding, and surrounding regions of the encoding gene using the same quality control criteria applied in the analysis of TTR variants. Additionally, we investigated the functional enrichments association related to the protein-protein interactions identified considering Gene Ontologies (Ashburner et al. 2000; The Gene Ontology 2019) for biological processes and molecular functions and molecular pathways available in the Reactome Database (Fabregat et al. 2018).

\section{Results}

The TTR PheWAS identified several phenotypic associations surviving FDR multiple testing correction (Fig. 1). These include associations identified in the overall analysis and in the sex-stratified analyses (Fig. 2, Supplementary File 3). In the analysis of the total cohort (Supplementary File 4), we observed several clinical signs that may be related to ATTRm and ATTRwt: spinal stenosis (FinnGen: M13_SPINSTENOSIS; rs116937108, beta $\left.=0.002, p=1.21 \times 10^{-7}\right)$, neuromuscular dysfunction of bladder (ICD-10: N31; rs9953311, beta $=-0.001, p=2.30 \times 10^{-6}$; rs575854233, beta $\left.=-0.001, p=5.33 \times 10^{-6}\right)$, and anxiety disorders (FINNGEN: KRA_PSY_ANXIETY; rs554521234, beta $\left.=0.003, p=8.85 \times 10^{-6}\right)$. Additionally, we also observed significant findings not expected to be directly related to TTR amyloidogenic process: Barret's esophagus (FinnGen: K11_BARRET; rs147570462, beta $=0.003$,

Table 1 Details regarding the clinically relevant traits and the genomic regions tested in the overall cohort and in the sex-stratified analyses

\begin{tabular}{llllll}
\hline Gene & $\begin{array}{l}\text { Chromosome Assembly } \\
\text { Localization }\end{array}$ & Sex & Phenotype $N$ & $\begin{array}{l}\text { Case median } N \text { (minimum- Control median } N(\text { mini- } \\
\text { maximum) }\end{array}$ & $\begin{array}{l}\text { mum-maximum) } \\
\text { mum }\end{array}$ \\
\hline TTR & NC_000018.9: & Both sexes & 382 & $2534(1011-208,009)$ & $358,660(79,185-360,183)$ \\
& $27,171,000-31,171,500$ & Female & 240 & $2364(1008-153,946)$ & $191,810(40,228-193,166)$ \\
& & Male & 225 & $2533(1004-128,063)$ & $164,487(39,957-166,016)$ \\
RBP4 & NC_000010.10: & Both sexes & $32^{\text {a }}$ & $2820(1014-20,857)$ & $358,374(340,337-360,180)$ \\
& $93,353,000-97,353,500$ & Female & $32^{\text {a }}$ & $1095(331-7355)$ & $193,079(186,819-193,843)$ \\
& & Male & $31^{\text {a }}$ & $1581(441-15,056)$ & $165,439(151,964-166,579)$ \\
\hline
\end{tabular}

We report the number of phenotypes tested and the sample size investigated in the overall-sample and sex-stratified analyses

${ }^{a}$ Significant phenotypic traits identified in the TTR PheWAS and tested in the RBP4 analysis 


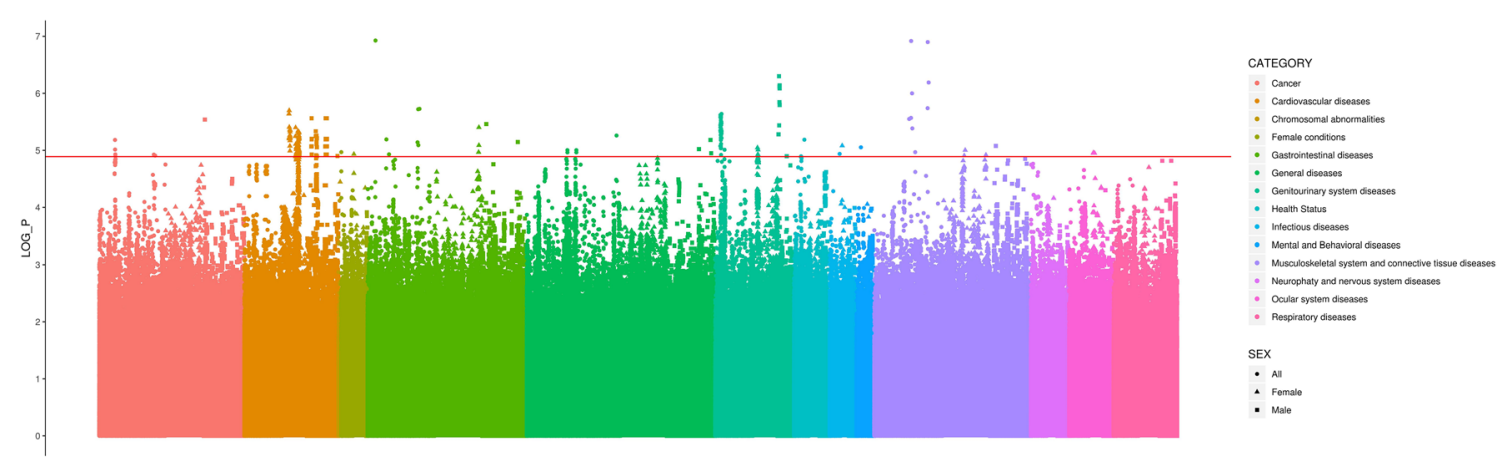

Fig. 1 Manhattan Plot of TTR PheWAS in the total sample and in sex-stratified analyses across the different phenotypic categories tested

$p=1.19 \times 10^{-7}$ ), abscess of anal and rectal regions (ICD10: $\mathrm{K} 61$; rs72948149, beta $\left.=0.002, p=1.87 \times 10^{-6}\right)$; fissure and fistula of anal and rectal regions (ICD-10: K60; rs72927361, beta $=-0.003, p=1.90 \times 10^{-6}$ ); complications of other internal prosthetic devices, implants and grafts (ICD-10: T85; rs34444556, beta $=0.003, p=5.5 \times 10^{-6}$ ), ulcer of esophagus (FinnGen: K11_OESULC; rs74481699, beta $\left.=0.004, p=6.43 \times 10^{-6}\right)$, adjustment and management of implanted device (ICD-10: Z45; rs117672477, beta $=0.002$, $\left.p=6.51 \times 10^{-6}\right)$; primary lymphoid and hematopoietic malignant neoplasms (FinnGen: C3_PRIMARY_LYMPHOID_HEMATOPOIETIC; rs 17718949, beta $=0.01$, $\left.p=6.57 \times 10^{-6}\right)$. Among the 240 clinically relevant phenotypes tested in the female-specific analysis $(N=194,174)$, we identified 8 LD-independent variants associated with specific pathological conditions (Supplementary File 5): chronic ischaemic heart disease (ICD-10: I25; rs140226130, beta $\left.=0.003, p=2.00 \times 10^{-6}\right)$, dysphagia $($ ICD-10: R13; rs2949506, beta $=0.002, p=3.95 \times 10^{-6}$ ), other disorders of urinary system (ICD-10: N39; rs71173870, beta $=0.003$, $p=9.81 \times 10^{-6}$ ), other specified/unspecified soft tissue disorders (ICD-10: M13_SOFTTISSUENAS; rs558461933, beta $\left.=0.007, p=9.92 \times 10^{-6}\right)$, other cataract $($ ICD-10: H26; rs 12966815 , beta $=0.003, p=1.10 \times 10^{-5}$ ), retinal detachments and breaks (ICD-10: H33; rs117637258, beta $=0.003, p=1.12 \times 10^{-5}$ ), other abnormal products of conception (ICD-10: O02; rs139327590, beta $=0.004$, $p=1.16 \times 10^{-5}$ ), and shoulder lesions (ICD-10: M75; rs77728273, beta $\left.=-0.004, p=1.21 \times 10^{-5}\right)$. Several of these clinical manifestations (e.g., I25 Chronic ischaemic heart disease, R13 Dysphagia, N39 Other disorders of urinary system, H26 Other cataract and H33 Retinal detachments and breaks) seem to be closely related to the expected symptoms of ATTRm and ATTRwt. The malespecific PheWAS (225 phenotypic traits tested) revealed several significant associations that could be related to cardiac, gastrointestinal, and urinary symptoms of ATTRm and ATTRwt (Supplementary File 6): other disorders of bladder (ICD-10: N32; rs 138038371, beta $\left.=0.009, p=5.01 \times 10^{-7}\right)$, heart failure (FinnGen: I9_HEARTFAIL; rs73956431, beta $\left.=0.005, p=2.74 \times 10^{-6}\right)$, malignant neoplasm of colon (ICD-10: C18; rs78431500, beta $=0.006, p=2.89 \times 10^{-6}$ ), Barret's esophagus (ICD-10: K11_BARRET; rs147570462, beta $\left.=0.005, p=3.45 \times 10^{-6}\right)$, atrial fibrillation and flutter (ICD-10: I48; rs10163755, beta $=0.003, p=4.63 \times 10^{-6}$ ), calculus of kidney and ureter (ICD-10: N20; rs8090264, beta $\left.=0.002, p=5.23 \times 10^{-6}\right)$, complications of procedures not elsewhere classified (ICD-10: T81; rs182526571, beta $\left.=0.008, p=6.57 \times 10^{-6}\right)$, other diseases of intestine (ICD-10: K63; rs970866, beta $=-0.005, p=7.14 \times 10^{-6}$ ) and fibroblastic disorders (FinnGen: M13_FIBROBLASTIC; rs60487427, beta $=-0.004, p=8.38 \times 10^{-6}$ ).

Amyloidogenic coding variants in TTR gene are the established cause of ATTRm (Conceicao 2012; Parman et al. 2016; Plante-Bordeneuve and Said 2011). The large sample size of the UK Biobank cohort includes carriers of TTR amyloidogenic mutations with high-quality genotype information (imputation info score $>0.8$; Supplementary File 7). However, due to the very low MAF of these coding mutations, the phenotypic associations of these variants should be considered only a "low-confidence" result as described in the methods. Being aware of this limitation, we explored their phenotypic spectrum in the UK Biobank (Table 2). The most relevant results were observed for the TTR Val122Ile mutation (rs76992529) in the female participants with respect to two well-known symptoms of the amyloidogenic process: carpal tunnel syndrome (FinnGen: G6_CARPTU; beta $\left.=0.307, p=6.41 \times 10^{-6}\right)$ and mononeuropathies of upper limb (ICD-10: G56; beta $=0.306, p=1.22 \times 10^{-5}$ ).

To test the presence of modifier genes involved in TTRrelated pathogenic processes, we investigated potential protein interaction and observed the highest-confidence interaction with respect to RBP4 protein, which is supported by experiments and co-expression interaction sources (STRING interaction score $=0.914$ ). The TTR$\mathrm{RBP} 4$ protein interaction is associated with functional enrichments for GO terms, including retinol metabolic process (GO:0042572, FDR $q=6.3 \times 10^{-4}$ ) and protein 
I25 (Chronic ischemic heart disease)

19_HEARTFAIL (Heart failure, strict)

148 (Atrial fibrillation and flutter)

R13 (Dysphagia)

K63 (Other diseases of intestine)

N31 (Neuromuscular dysfunction of bladder, not elsewhere classified)

N39 (Other disorders of urinary system)

N32 (Other disorders of bladder)

N20 (Calculus of kidney and ureter)

H26 (Other cataract)

H33 (Retinal detachments and breaks)

M13 (SPINSTENOSIS (Spinal stenosis)

KRA_PSY_ANXIETY (Anxiety disorders)

M13_FIBROBLASTIC (Fibroblastic disorders)

M13_SOFTTISSUES (Other specified/unspecified soft tissue disorders)

M75 (Shoulder lesions)

K61 (Abscess of al and rectal regions)

$\mathrm{K} 60$ (Fissure and fistula of al and rectal regions)

K11_BARRET (Barret esophagus)

K11_OESULC (Ulcer of esophagus)

C18 (Malign neoplasm of colon)

C3_PRIMARY_LYMPHOID_HEMATOPOIETIC (Primary lymphoid and hematopoietic malign neoplasms

T85 (Complications of other internal prosthetic devices, implants and grafts)

T81 (Complications of procedures, not elsewhere classified)

Z45 (Adjustment and management of implanted device)

$\mathrm{O} 2$ (Other abnormal products of conception)

$-6$

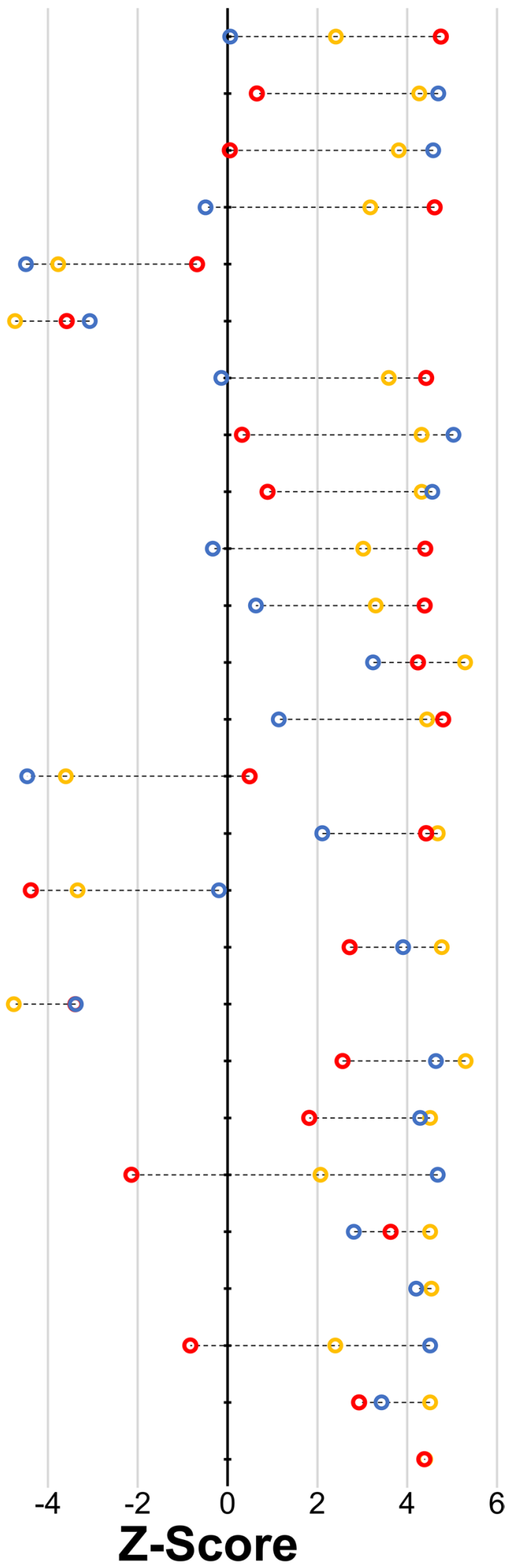

(Overall,Female,Male)

Fig. 2 Associations (Z-Scores) of non-coding variants surviving multiple testing correction in the TTR PheWAS in the overall-sample and sexstratified analyses. Details of the associations are reported in Supplementary File 3 
Table 2 Significant associations identified with respect to TTR coding mutations

\begin{tabular}{|c|c|c|c|c|c|c|}
\hline Phenotypic trait & rsid & Substitution & Beta & SE & $P$ value & FDR \\
\hline \multicolumn{7}{|l|}{ Both sexes } \\
\hline $\begin{array}{l}\text { FinnGen: XVII_MALFORMAT_ABNORMAL Congenital malforma- } \\
\text { tions, deformations and chromosomal abnormalities }\end{array}$ & rs138657343 & $\underline{\operatorname{Arg} 5 \mathrm{His}}$ & 0.0264 & 0.00550 & $1.64 \times 10^{-6}$ & 0.029 \\
\hline \multicolumn{7}{|l|}{ Female } \\
\hline ICD10: R87 Abnormal findings in specimens from female genital organs & rs 138657343 & $\underline{\operatorname{Arg} 5 \mathrm{His}}$ & 0.0379 & 0.00724 & $1.65 \times 10^{-7}$ & 0.004 \\
\hline $\begin{array}{l}\text { FinnGen: XVII_MALFORMAT_ABNORMAL Congenital malforma- } \\
\text { tions, deformations and chromosomal abnormalities }\end{array}$ & & & 0.0368 & 0.00756 & $1.12 \times 10^{-6}$ & 0.019 \\
\hline $\begin{array}{l}\text { ICD10: N94 Pain and other conditions associated with female genital } \\
\text { organs and menstrual cycle }\end{array}$ & rs76992529 & Val122Ile & 0.158 & 0.0334 & $2.31 \times 10^{-6}$ & 0.032 \\
\hline FinnGen: G6_CARPTU Carpal tunnel syndrome & & & 0.307 & 0.0681 & $6.41 \times 10^{-6}$ & 0.063 \\
\hline ICD10: G56 Mononeuropathies of upper limb & & & 0.306 & 0.0699 & $1.22 \times 10^{-5}$ & 0.079 \\
\hline FinnGen: K11_HERNIA Hernia & rs121918074 & His90Asn & 0.210 & 0.0485 & $1.53 \times 10^{-5}$ & 0.090 \\
\hline
\end{tabular}

Information about beta value, standard error (SE), $p$ value and false discovery rate (FDR) $q$ value are reported. Bold text: TTR protein substitution. Underline text: TTR precursor substitution. Information about allele frequency and minor allele frequency are reported in Supplementary File 7

heterodimerization activity (GO:0046982, FDR $q=0.02$ ), and for Reactome molecular pathways, including retinol cycle disease events (HSA-2453864, FDR $q=9.98 \times 10^{-6}$ ), canonical retinoid cycle in rods (HSA-2453902, FDR $\left.q=1.1 \times 10^{-5}\right)$, and retinoid metabolism and transport (HSA-975634, FDR $q=2.84 \times 10^{-5}$ ). On the basis of these data, we hypothesize a convergence where variants located in TTR and RBP4 genes are associated with clinically relevant phenotypes related to ATTRm and ATTRwt pathogenesis. Accordingly, we investigated 13,226, 13,231, and 13217 high-confidence variants (overall-sample, femalespecific, and male-specific analyses, respectively) located in $R B P 4$ gene and its surrounding regions (NC_000010.10: 93,353,000-97,353,500) with respect to the significant phenotypic traits observed in the TTR PheWAS (Table 1). We identified significant associations in the overall cohort and in the sex-stratified analyses (Supplementary File 3). Considering the traits tested across different phenotypic categories, we observed a concordant trend between TTR and RBP4 associations observed in the overall sample and in the sexstratified analyses (Fig. 3). In the RBP4 analysis conducted in the overall sample, we confirmed some of the TTR associations previously observed with respect to TTR gene that are related to known clinical signs of ATTRm and ATTRwt (Supplementary File 8): heart failure (FinnGen: I9_HEARTFAIL; rs1326222, beta $=0.001, p=5.25 \times 10^{-7}$ ) and anxiety disorders (FinnGen: KRA_PSY_ANXIETY; rs112059561, beta $\left.=0.002, p=6.44 \times 10^{-6}\right)$. Among the phenotypes that do not seem to be related to TTR amyloidogenic processes, Barret's esophagus was also significantly associated with a RBP4 variant (FinnGen: K11_BARRET; rs12573026, beta $\left.=0.001, p=9.96 \times 10^{-8}\right)$. In the female-specific analysis, we observed significant associations with respect to cardiac symptoms which are one of the leading signs of ATTRm and ATTRwt: atrial fibrillation and flutter (ICD10: I48; rs4917692, beta $=0.002, p=1.15 \times 10^{-5}$ ) and heart
Fig. 3 Association of $R B P 4$ common genetic variants with traits surviving multiple testing correction in the TTR PheWAS in the overall-sample and sex-stratified analyses. Red dotted lines represent nominal significance $(p<0.05)$

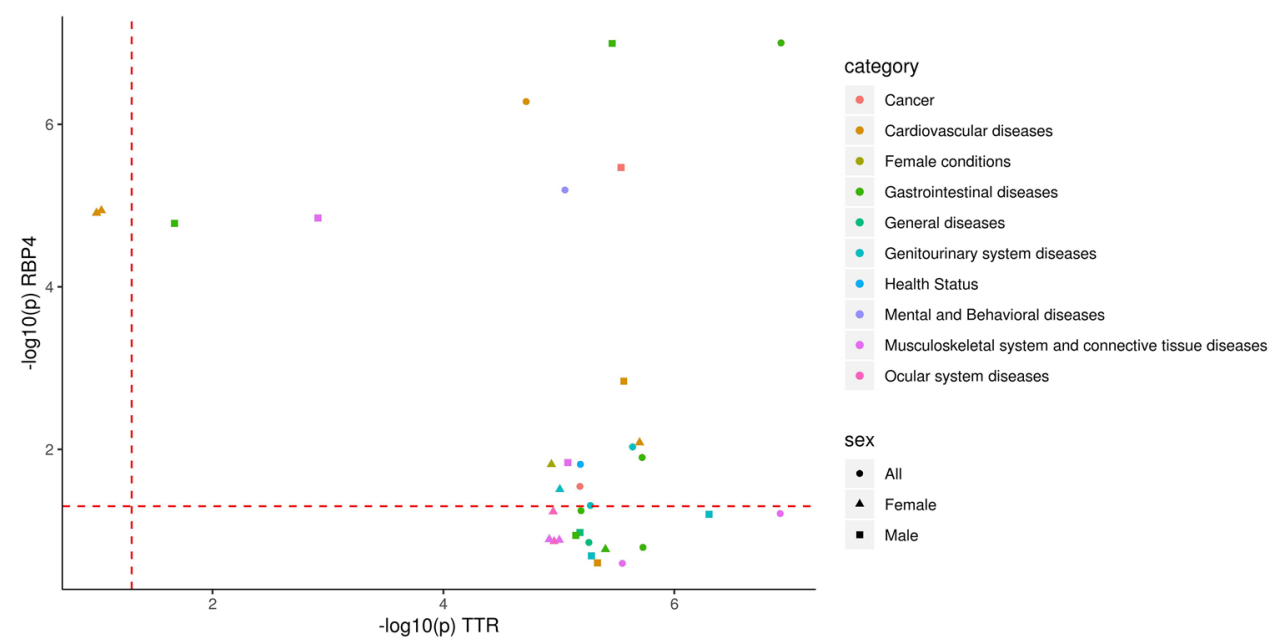


failure (FinnGen: I9_HEARTFAIL; rs1326222, beta $=0.001$, $\left.p=1.23 \times 10^{-5}\right)($ Supplementary File 9$)$. Finally, in the male participants, we observed a convergence between $R B P 4$ and TTR findings with respect to two additional phenotypes expected to be related to ATTRm and ATTRwt (FinnGen: M13_SPINSTENOSIS spinal stenosis, rs112288944, beta $=0.005, p=1.42 \times 10^{-5}$; ICD-10: R13 $\sim$ Dysphagia, rs61886346, beta $=0.003, p=1.66 \times 10^{-5}$ ). Some conditions that are not expected to be linked to amyloidogenic processes such as Barret's esophagus (FinnGen: K11_ BARRET; rs12573026, beta $\left.=0.002, p=1.01 \times 10^{-7}\right)$ and malignant neoplasm of colon (ICD-10: C18; rs142083973, beta $=0.005, p=3.40 \times 10^{-6}$ ) were also associated with both RBP4 and TTR gene variants in the male sample (Supplementary File 10). Supplementary File 11 summarizes all significant associations, providing the allele frequencies of $T T R$ and $R B P 4$ variants identified.

\section{Discussion}

TTR misfolding and the following amyloid formation and deposition are the cause of ATTRm and ATTRwt due to TTR coding variants and the misfolding of the wild type protein, respectively (Plante-Bordeneuve and Said 2011; Westermark et al. 1990). With respect to ATTRm, several studies investigated the role of TTR coding and non-coding variation to dissect the molecular machineries at the basis of its complex phenotype-genotype correlation (Iorio et al. 2015, 2017a, b; Polimanti et al. 2013, 2014, 2019; Sikora et al. 2015). Differently, limited information is available regarding the genetic basis of ATTRwt (Sikora et al. 2015). PheWAS design is a powerful tool to broaden the knowledge about the phenotypic spectrum associated with disease-causing genetic variations (Bush et al. 2016; Denny et al. 2013; Polimanti et al. 2016) and its performance is enhanced by the availability of genomic data of large cohorts. Accordingly, we conducted a phenome-wide investigation of TTR coding and non-coding variants in more than 300,000 participants of European descent, also evaluating the RBP4 gene as a possible modifier of TTR pathogenetic mechanisms.

Our results pointed out novel associations of TTR noncoding variants with phenotypic conditions potentially related to ATTRm and ATTRwt pathogenesis. In the sex stratified analysis, we observed strong evidence of the effect of TTR non-coding variations on cardiac involvement (ICD-10: I25 Chronic ischaemic heart disease; I48 Atrial fibrillation and flutter; FinnGen: I9_HEARTFAIL Heart failure, strict), one of the leading clinical signs of ATTRm and ATTRwt. With respect to ATTRwt, heart is the most affected organ in elderly patients, and, the main manifestations are cardiomyopathy (resulting in increase of biventricular wall thickness and ventricular stiffness) and atrial fibrillation (Ando et al. 2013; Siddiqi and Ruberg 2018). The symptoms associated with ATTRm include a restrictive amyloid cardiomyopathy along with a combination of several other signs, including gait, gastrointestinal, neurological, urinary/renal, and ocular involvement frequently reported among the affected carriers (Ando et al. 2013; Parman et al. 2016; Plante-Bordeneuve and Said 2011; Siddiqi and Ruberg 2018).

GI symptoms were identified in our phenome-wide investigation (ICD-10: R13 Dysphagia and K63 Other diseases of intestine). The occurrence of GI manifestations has been reported in both ATTRm and ATTRwt and these include nausea, vomiting, constipation, faecal incontinence, and weight loss (Ando et al. 2013; Collins et al. 2018; Wixner et al. 2014). Although GI involvement is reported in ATTRwt patients, these symptoms are less common than the ones reported in ATTRm (Wixner et al. 2014), where GI manifestations are more frequent in earlyonset patients (Wixner et al. 2014).

Significant associations of $T T R$ variants with respect to bladder, urinary tract and kidney were identified in the overall sample and in the sex-stratified analyses (ICD-10: N31 Neuromuscular dysfunction of bladder, not elsewhere classified; N39 Other disorders of urinary system; N32 Other disorders of bladder; N20 Calculus of kidney and ureter). It is well known the evolution of neurogenic bladder in ATTRm that is linked to autonomic nerve dysfunction, and, renal involvement due to the amyloid deposition in the glomeruli arterioles and medium vessels (Ando et al. 2013; Lobato and Rocha 2012).

The female sample showed that TTR non-coding variants are associated with ocular involvement (ICD-10: H26 Other cataract; H33 Retinal detachments and breaks). These findings are consistent with the known ocular manifestations described in ATTRm, which, in agreement with our sex-stratified result, occur more frequently in women (Reynolds et al. 2017). The ocular symptoms reported in ATTRm patients include vitreous opacities, retinal vein occlusion and direct optic nerve infiltration (Reynolds et al. 2017).

Remarkably, a significant genetic association between TTR gene and anxiety disorders (FinnGen: KRA_PSY_ ANXIETY) was observed. There is a growing literature regarding the effect of rare life-threatening diseases on the mental health of the patients and their families. With respect to ATTRm, several studies reported considerable psychological consequences in carriers of TTR mutations after the onset of the symptoms and the diagnosis of the disease (Graceffa et al. 2009; Lopes et al. 2018). These behavioural changes seem to be related to the stressful scenarios related to amyloidosis diagnostic path, including the pre-symptomatic genetic testing confirming the presence of an amyloidogenic mutation in family members and the time (usually 
years) intervening between the onset of the symptoms and the diagnosis of the diseases in novel ATTRm cases.

Spinal stenosis (FinnGen: M13_SPINSTENOSIS) was another phenotype identified in our TTR Phewas. This symptom has been reported in both ATTRm and ATTRwt patients (Ando et al. 2013; Siddiqi and Ruberg 2018), and it usually co-occurs with other known signs of TTR amyloidogenic process such as carpal tunnel syndrome and neuropathies (Carr et al. 2019). These phenotypic traits were identified as associated to TTR Val122Ile mutation (Table 2). This is also consistent with phenotypic presentation of Val122Ile carriers affected by ATTRm where the carpal tunnel syndrome represents an early sign of the disease whereas upper limb involvement is a clinical manifestation observed in a later stage (Ando et al. 2013). Although our findings are related to carriers of European descent and Val122Ile mutation is mainly identified in individuals of African descent, a similar clinical phenotype has been reported in Val122Ile carriers of both ancestry groups (Cappelli et al. 2016).

Our findings support a phenotypic convergence between $T T R$ and RBP4 genetic associations, also including traits expected to be associated with ATTRm and ATTRwt clinical spectrum (i.e., heart failure and atrial fibrillation). This evidence supports the putative role of RBP4 as modifier gene with respect to TTR amyloidogenic process. RBP4 interacts with TTR via formation of TTR-RBP4 protein complex that stabilizes the TTR tetramer, inhibiting monomer dissociation and fibril formation (Palaninathan 2012). In carriers of TTR amyloidogenic mutations, there is an increase of the TTR tetramer dissociation with a consequent decrease of the TTR-RBP4 complex and an increase of the RBP4 urinary excretion (Palaninathan 2012; Arvanitis et al. 2017). Accordingly, RBP4 genetic variation could affect the functionality of the TTR-RBP4 complex, contributing to the complex phenotype-genotype correlation in carriers of TTR amyloidogenic mutations and increasing the risk of ATTRwt in non-carriers.

Although the findings presented provided an unprecedented amount of information regarding the phenotypic spectrum associated with common genetic variants located in TTR gene and its surrounding region, our study present several limitations. UK biobank is an amazing resource to investigate the molecular bases of human diseases. However, the depth of the phenotypic characterization available in the UK Biobank at this time does not permit to fully characterize certain aspects related to ATTR pathogenesis. For instance, we observed significant association of TTR variants with heart failure. However, ATTR is associated with heart failure with preserved ejection fraction. Further studies will be needed to verify common variants in TTR gene are associated with the same cardiac phenotype observed in ATTR patients. Another limitation is that the UK Biobank cohort includes subjects representative of the general population that were not recruited specifically for ATTR research. Accordingly, several of the phenotypic association observed appear to be unrelated to ATTR pathogenesis. These traits can potentially be related to the inter-individual variability of the main physiological function of TTR and RBP4 protein products: the transport of retinol (vitamin A) (Noy 2016). Altered homeostasis of vitamin is associated with several disorders (Mason et al. 2015) and some of them could be associated with the effect of TTR and RBP4 variants on retinol metabolism, explaining some of the phenotypic traits identified in the present study.

In conclusion, the present study provides novel insights about TTR gene variation, confirming its role in phenotypic variability observed in ATTRm patients and supporting its potential involvement in the predisposition to ATTRwt. These data support the relevance of large biobanks to investigate complex genotype-phenotype associations. Additionally, the insights provided support the necessity of further investigations of TTR non-coding variation together with putative modifier loci to develop tools able to anticipate the course of the disease to improve the diagnosis and management of patients affected by ATTRm and ATTRwt.

Acknowledgements We thank the participants and investigators of the UK Biobank and the Neale lab for generating the genome-wide data used in the present study. This work was partially supported by the $\mathrm{PhD}$ Program of the University of Rome Tor Vergata (ADL) and the Yale University School of Medicine (RP). The sources of funding had no role in the design of the study and collection, analysis, and interpretation of data and in writing the manuscript.

Data availability Data supporting the findings of this study are available within this article and its additional files. UK Biobank GWAS summary association data are available at https://github.com/Nealelab/ UK_Biobank_GWAS/tree/master/imputed-v2-gwas.

\section{Compliance with ethical standards}

Conflict of interest Drs. Fuciarelli and Polimanti are both receiving research grants from Pfizer Inc. to conduct epigenetic studies of TTR amyloidosis. The other authors reported no biomedical financial interests or potential conflicts of interest.

Ethical approval This study was conducted using summary association data generated by previous studies. Owing to the use of previously collected, deidentified, aggregated data, this study did not require institutional review board approval.

\section{References}

Allen NE, Sudlow C, Peakman T, Collins R, Biobank UK (2014) UK biobank data: come and get it. Sci Transl Med 6: 224ed4. https:// doi.org/10.1126/scitranslmed.3008601

Alves-Ferreira M, Coelho T, Santos D, Sequeiros J, Alonso I, Sousa A, Lemos C (2018) A trans-acting factor may modify age at onset in familial amyloid polyneuropathy ATTRV30M in Portugal. 
Mol Neurobiol 55:3676-3683. https://doi.org/10.1007/s1203 5-017-0593-4

Ando Y, Coelho T, Berk JL, Cruz MW, Ericzon BG, Ikeda S, Lewis WD, Obici L, Plante-Bordeneuve V, Rapezzi C, Said G, Salvi F (2013) Guideline of transthyretin-related hereditary amyloidosis for clinicians. Orphanet J Rare Dis 8:31. https://doi. org/10.1186/1750-1172-8-31

Arvanitis M, Koch CM, Chan GG, Torres-Arancivia C, LaValley MP, Jacobson DR, Berk JL, Connors LH, Ruberg FL (2017) Identification of transthyretin cardiac amyloidosis using serum retinolbinding protein 4 and a clinical prediction model. JAMA Cardiol 2:305-313. https://doi.org/10.1001/jamacardio.2016.5864

Ashburner M, Ball CA, Blake JA, Botstein D, Butler H, Cherry JM, Davis AP, Dolinski K, Dwight SS, Eppig JT, Harris MA, Hill DP, Issel-Tarver L, Kasarskis A, Lewis S, Matese JC, Richardson JE, Ringwald M, Rubin GM, Sherlock G (2000) Gene ontology: tool for the unification of biology. The Gene Ontology Consortium. Nat Genet 25:25-29. https://doi.org/10.1038/75556

Benjamini Y, Hochberg Y (1995) Controlling the false discovery rate: a practical and powerful approach to multiple testing. J Roy Stat Soc Ser B (Methodol) 57:289-300

Bush WS, Oetjens MT, Crawford DC (2016) Unravelling the human genome-phenome relationship using phenome-wide association studies. Nat Rev Genet 17:129-145. https://doi.org/10.1038/ nrg. 2015.36

Bycroft C, Freeman C, Petkova D, Band G, Elliott LT, Sharp K, Motyer A, Vukcevic D, Delaneau O, O'Connell J, Cortes A, Welsh S, Young A, Effingham M, McVean G, Leslie S, Allen N, Donnelly P, Marchini J (2018) The UK Biobank resource with deep phenotyping and genomic data. Nature 562:203-209. https://doi. org/10.1038/s41586-018-0579-z

Cappelli F, Frusconi S, Bergesio F, Grifoni E, Fabbri A, Giuliani C, Falconi S, Bonifacio S, Perfetto F (2016) The Val142Ile transthyretin cardiac amyloidosis: not only an Afro-American pathogenic variant? A single-centre Italian experience. J Cardiovasc Med (Hagerstown) 17:122-125. https://doi.org/10.2459/ JCM.0000000000000290

Carr AS, Shah S, Choi D, Blake J, Phadke R, Gilbertson J, Whelan CJ, Wechalekar AD, Gillmore JD, Hawkins PN, Reilly MM (2019) Spinal stenosis in familial transthyretin amyloidosis. J Neuromuscul Dis 6:267-270. https://doi.org/10.3233/JND-180348

Chang CC, Chow CC, Tellier LC, Vattikuti S, Purcell SM, Lee JJ (2015) Second-generation PLINK: rising to the challenge of larger and richer datasets. Gigascience 4:7. https://doi.org/10.1186/ s13742-015-0047-8

Collins M, Pellat A, Antoni G, Agostini H, Labeyrie C, Adams D, Carbonnel F (2018) Somatostatin analogues for refractory diarrhoea in familial amyloid polyneuropathy. PLoS One 13:e0201869. https ://doi.org/10.1371/journal.pone.0201869

Conceicao I (2012) Clinical features of TTR-FAP in Portugal. Amyloid 19(Suppl 1):71-72. https://doi.org/10.3109/13506129.2012.67318 4

Conceicao I, Damy T, Romero M, Galan L, Attarian S, Luigetti M, Sadeh M, Sarafov S, Tournev I, Ueda M (2019) Early diagnosis of ATTR amyloidosis through targeted follow-up of identified carriers of TTR gene mutations. Amyloid 26:3-9. https://doi. org/10.1080/13506129.2018.1556156

Connors LH, Doros G, Sam F, Badiee A, Seldin DC, Skinner M (2011) Clinical features and survival in senile systemic amyloidosis: comparison to familial transthyretin cardiomyopathy. Amyloid 18(Suppl 1):157-159. https://doi.org/10.3109/13506 129.2011.574354059

Denny JC (2012) Chapter 13: mining electronic health records in the genomics era. PLoS Comput Biol 8: e1002823. https://doi. org/10.1371/journal.pcbi.1002823
Denny JC, Bastarache L, Ritchie MD, Carroll RJ, Zink R, Mosley JD, Field JR, Pulley JM, Ramirez AH, Bowton E, Basford MA, Carrell DS, Peissig PL, Kho AN, Pacheco JA, Rasmussen LV, Crosslin DR, Crane PK, Pathak J, Bielinski SJ, Pendergrass SA, $\mathrm{Xu}$ H, Hindorff LA, Li R, Manolio TA, Chute CG, Chisholm RL, Larson EB, Jarvik GP, Brilliant MH, McCarty CA, Kullo IJ, Haines JL, Crawford DC, Masys DR, Roden DM (2013) Systematic comparison of phenome-wide association study of electronic medical record data and genome-wide association study data. Nat Biotechnol 31:1102-1110. https://doi.org/10.1038/nbt.2749

Fabregat A, Jupe S, Matthews L, Sidiropoulos K, Gillespie M, Garapati P, Haw R, Jassal B, Korninger F, May B, Milacic M, Roca CD, Rothfels K, Sevilla C, Shamovsky V, Shorser S, Varusai T, Viteri G, Weiser J, Wu G, Stein L, Hermjakob H, D'Eustachio P (2018) The reactome pathway knowledgebase. Nucleic Acids Res 46:D649-D655. https://doi.org/10.1093/nar/gkx1132

Graceffa A, Russo M, Vita GL, Toscano A, Dattola R, Messina C, Vita G, Mazzeo A (2009) Psychosocial impact of presymptomatic genetic testing for transthyretin amyloidotic polyneuropathy. Neuromuscul Disord 19:44-48. https://doi.org/10.1016/j. nmd.2008.09.017

Hellman U, Alarcon F, Lundgren HE, Suhr OB, Bonaiti-Pellie C, Plante-Bordeneuve V (2008) Heterogeneity of penetrance in familial amyloid polyneuropathy, ATTR Val30 Met, in the Swedish population. Amyloid 15:181-186. https://doi. org/10.1080/13506120802193720

Iorio A, De Angelis F, Di Girolamo M, Luigetti M, Pradotto L, Mauro A, Manfellotto D, Fuciarelli M, Polimanti R (2015) Most recent common ancestor of TTR Val30 Met mutation in Italian population and its potential role in genotype-phenotype correlation. Amyloid 22:73-78. https://doi.org/10.3109/13506129.2014.99459 7

Iorio A, De Angelis F, Di Girolamo M, Luigetti M, Pradotto LG, Mazzeo A, Frusconi S, My F, Manfellotto D, Fuciarelli M, Polimanti R (2017a) Population diversity of the genetically determined TTR expression in human tissues and its implications in TTR amyloidosis. BMC Genomics 18:254. https://doi.org/10.1186/ s12864-017-3646-1

Iorio A, De Lillo A, De Angelis F, Di Girolamo M, Luigetti M, Sabatelli M, Pradotto L, Mauro A, Mazzeo A, Stancanelli C, Perfetto F, Frusconi S, My F, Manfellotto D, Fuciarelli M, Polimanti R (2017b) Non-coding variants contribute to the clinical heterogeneity of TTR amyloidosis. Eur J Hum Genet 25:1055-1060. https:// doi.org/10.1038/ejhg.2017.95

Kyle RA, Gertz MA (1995) Primary systemic amyloidosis: clinical and laboratory features in 474 cases. Semin Hematol 32:45-59

Lobato L, Rocha A (2012) Transthyretin amyloidosis and the kidney. Clin J Am Soc Nephrol 7:1337-1346. https://doi.org/10.2215/ CJN.08720811

Lopes A, Sousa A, Fonseca I, Branco M, Rodrigues C, Coelho T, Sequeiros J, Freitas P (2018) Life paths of patients with transthyretin-related familial amyloid polyneuropathy Val30 Met: a descriptive study. J Community Genet 9:93-99. https://doi. org/10.1007/s12687-017-0338-0

Maceira AM, Joshi J, Prasad SK, Moon JC, Perugini E, Harding I, Sheppard MN, Poole-Wilson PA, Hawkins PN, Pennell DJ (2005) Cardiovascular magnetic resonance in cardiac amyloidosis. Circulation 111:186-193. https://doi.org/10.1161/01.CIR.0000152819 97857.9D

Mason J, Greiner T, Shrimpton R, Sanders D, Yukich J (2015) Vitamin A policies need rethinking. Int J Epidemiol 44:283-292. https:// doi.org/10.1093/ije/dyu194

Nakagawa M, Sekijima Y, Yazaki M, Tojo K, Yoshinaga T, Doden T, Koyama J, Yanagisawa S, Ikeda S (2016) Carpal tunnel syndrome: a common initial symptom of systemic wild-type 
ATTR (ATTRwt) amyloidosis. Amyloid 23:58-63. https://doi. org/10.3109/13506129.2015.1135792

Noy N (2016) Vitamin A in regulation of insulin responsiveness: mini review. Proc Nutr Soc 75:212-215. https://doi.org/10.1017/S0029 665115004322

Palaninathan SK (2012) Nearly 200 X-ray crystal structures of transthyretin: what do they tell us about this protein and the design of drugs for TTR amyloidoses? Curr Med Chem 19:2324-2342

Parman Y, Adams D, Obici L, Galan L, Guergueltcheva V, Suhr OB, Coelho T, European Network for T-F (2016) Sixty years of transthyretin familial amyloid polyneuropathy (TTR-FAP) in Europe: where are we now? A European network approach to defining the epidemiology and management patterns for TTR-FAP. Curr Opin Neurol 29(Suppl 1):S3-S13. https://doi.org/10.1097/WCO.00000 00000000288

Pitkanen P, Westermark P, Cornwell GG 3rd (1984) Senile systemic amyloidosis. Am J Pathol 117:391-399

Plante-Bordeneuve V, Said G (2011) Familial amyloid polyneuropathy. Lancet Neurol 10:1086-1097. https://doi.org/10.1016/S1474 $-4422(11) 70246-0$

Polimanti R, Di Girolamo M, Manfellotto D, Fuciarelli M (2013) Functional variation of the transthyretin gene among human populations and its correlation with amyloidosis phenotypes. Amyloid 20:256-262. https://doi.org/10.3109/13506129.2013.844689

Polimanti R, Di Girolamo M, Manfellotto D, Fuciarelli M (2014) In silico analysis of TTR gene (coding and non-coding regions, and interactive network) and its implications in transthyretin-related amyloidosis. Amyloid 21:154-162. https://doi.org/10.3109/13506 129.2014.900487

Polimanti R, Kranzler HR, Gelernter J (2016) Phenome-wide association study for alcohol and nicotine risk alleles in 26394 women. Neuropsychopharmacology 41:2688-2696. https://doi. org/10.1038/npp.2016.72

Polimanti R, Nunez YZ, Gelernter J (2019) Increased risk of multiple outpatient surgeries in African-American carriers of transthyretin Val122Ile mutation is modulated by non-coding variants. J Clin Med. https://doi.org/10.3390/jcm8020269

Reynolds MM, Veverka KK, Gertz MA, Dispenzieri A, Zeldenrust SR, Leung N, Pulido JS (2017) Ocular manifestations of familial transthyretin amyloidosis. Am J Ophthalmol 183:156-162. https ://doi.org/10.1016/j.ajo.2017.09.001

Santos D, Coelho T, Alves-Ferreira M, Sequeiros J, Mendonca D, Alonso I, Lemos C, Sousa A (2016) Variants in RBP4 and AR genes modulate age at onset in familial amyloid polyneuropathy (FAP ATTRV30M). Eur J Hum Genet 24:756-760. https://doi. org/10.1038/ejhg.2015.180
Siddiqi OK, Ruberg FL (2018) Cardiac amyloidosis: an update on pathophysiology, diagnosis, and treatment. Trends Cardiovasc Med 28:10-21. https://doi.org/10.1016/j.tcm.2017.07.004

Sikora JL, Logue MW, Chan GG, Spencer BH, Prokaeva TB, Baldwin CT, Seldin DC, Connors LH (2015) Genetic variation of the transthyretin gene in wild-type transthyretin amyloidosis (ATTRwt). Hum Genet 134:111-121. https://doi.org/10.1007/ s00439-014-1499-0

Soares ML, Coelho T, Sousa A, Batalov S, Conceicao I, Sales-Luis ML, Ritchie MD, Williams SM, Nievergelt CM, Schork NJ, Saraiva MJ, Buxbaum JN (2005) Susceptibility and modifier genes in Portuguese transthyretin V30M amyloid polyneuropathy: complexity in a single-gene disease. Hum Mol Genet 14:543-553. https://doi.org/10.1093/hmg/ddi051

Szklarczyk D, Gable AL, Lyon D, Junge A, Wyder S, Huerta-Cepas J, Simonovic M, Doncheva NT, Morris JH, Bork P, Jensen LJ, Mering CV (2019) STRING v11: protein-protein association networks with increased coverage, supporting functional discovery in genome-wide experimental datasets. Nucleic Acids Res 47:D607D613. https://doi.org/10.1093/nar/gky1131

The Gene Ontology C (2019) The Gene Ontology Resource: 20 years and still GOing strong. Nucleic Acids Res 47:D330-D338. https ://doi.org/10.1093/nar/gky1055

Wei WQ, Denny JC (2015) Extracting research-quality phenotypes from electronic health records to support precision medicine. Genome Med 7:41. https://doi.org/10.1186/s13073-015-0166-y

Westermark P, Sletten K, Johansson B, Cornwell GG 3rd (1990) Fibril in senile systemic amyloidosis is derived from normal transthyretin. Proc Natl Acad Sci USA 87:2843-2845. https://doi. org/10.1073/pnas.87.7.2843

White JT, Kelly JW (2001) Support for the multigenic hypothesis of amyloidosis: the binding stoichiometry of retinol-binding protein, vitamin $\mathrm{A}$, and thyroid hormone influences transthyretin amyloidogenicity in vitro. Proc Natl Acad Sci USA 98:13019-13024. https://doi.org/10.1073/pnas.241406698

Wixner J, Mundayat R, Karayal ON, Anan I, Karling P, Suhr OB, investigators T (2014) THAOS: gastrointestinal manifestations of transthyretin amyloidosis - common complications of a rare disease. Orphanet J Rare Dis 9:61. https://doi.org/10.1186/1750-1172-9-61

Publisher's Note Springer Nature remains neutral with regard to jurisdictional claims in published maps and institutional affiliations. 